\title{
The Buckland Park MF radar: routine observation scheme and velocity comparisons
}

\author{
D. A. Holdsworth ${ }^{1,2}$ and I. M. Reid ${ }^{2}$ \\ ${ }^{1}$ Atmospheric Radar Systems, 1/26 Stirling St, Thebarton, South Australia, Australia \\ ${ }^{2}$ Department of Physics and Mathematical Physics, The University of Adelaide, South Australia, Australia \\ Received: 1 October 2003 - Revised: 18 December 2003 - Accepted: 9 January 2004 - Published: 29 November 2004 \\ Part of Special Issue "10th International Workshop on Technical and Scientific Aspects of MST Radar (MST10)"
}

\begin{abstract}
This paper describes the routine observations scheme implemented for the Buckland Park medium frequency (BPMF) radar. These observations are rare among current MF/HF radar observations in that they are made using a relatively narrow transmit polar diagram. The flexibility of the radar allows a number of analyses to be performed simultaneously. The analyses described include the full correlation analysis (FCA), spatial correlation analysis (SCA), hybrid Doppler interferometry (HDI) and imaging Doppler interferometry (IDI) for observations of mesospheric dynamics and the temporal and spatial characteristics of their scatterers, the differential absorption experiment (DAE) for the estimation of electron densities and collision frequencies, and meteor analysis for estimation of meteor height, time and angle of arrival (AOA) distributions. Intercomparisons between wind velocities estimated using the FCA with SCA, HDI and IDI techniques are presented. The FCA velocities exhibit the well-known "triangle size effect" (TSE), whereby the wind velocity is underestimated at smaller antenna spacings. Although the SCA, IDI and HDI techniques were not applied concurrently, comparisons using FCA as a reference suggest these techniques produce velocities in good agreement.
\end{abstract}

Key words. Ionosphere (instruments and techniques) - Meteorology and atmospheric dynamics (instruments and techniques) - Radio science (instruments and techniques)

\section{Introduction}

Medium (MF) and high frequency (HF) radars are capable of providing continuous height coverage between 60 and $100 \mathrm{~km}$ during the day and 80 and $100 \mathrm{~km}$ at night (e.g. Hocking, 1997b). Although MF/HF radars have limited range resolution due to the relatively long wavelengths used (e.g. $\lambda=150 \mathrm{~m}$ at $2 \mathrm{MHz}$ ), they are ideal for routine observations, allowing continuous measurements of winds for es-

Correspondence to: D. A. Holdsworth

(dholdswo@atrad.com.au) timation of waves, tides and turbulence. The majority of $\mathrm{MF} / \mathrm{HF}$ radars used for routine observations are "spaced antenna" (SA) systems, consisting of a small transmitting array and a number of spaced antennas for reception (e.g. Vincent and Lesicar, 1991).

Until recently, routine spaced antenna observations made using the Buckland Park MF (BPMF) radar were carried out in a similar manner. The original radar used a small transmitting array and a large 1-km diameter array for reception. Routine spaced antenna observations were performed using reception on three groups of four antennas arranged in an almost equilateral triangle. The BPMF radar was overhauled between 1991 and 1995, involving the replacement of the main antenna array and the transmitting and receiving systems (e.g. Reid et al., 1995). The large array can now be used for both transmission and reception, allowing for the use of relatively narrow transmit beams (e.g. Vandepeer and Reid, 1995). This provides significant advantages over typical $\mathrm{MF} / \mathrm{HF}$ radar systems, in that more power is directed vertically, where the majority of power is reflected from the aspect sensitive scatterers observed at MF/HF (e.g. Lesicar and Hocking, 1992a).

The flexibility of the improved BPMF radar allows for a number of routine analyses to be performed simultaneously. This paper describes the routine observations scheme and presents sample results, thereby extending Reid et al. (1995), who presented the radar specifications. Section 2 describes the radar system, the experimental configuration based on an evaluation of the antenna and receiver characteristics of the system, and the implementation of the routine analyses. Sections 3 to 8 describe the routine analyses and sample results from the routine analyses, while Sect. 9 presents sample velocity comparisons. Results from specific analyses are presented in more detail in Holdsworth and Reid (1997), Holdsworth et al. (2001), Holdsworth et al. (2002), Vuthaluru et al. (2002), Holdsworth and Reid (2004), and in a number of papers currently in preparation. 

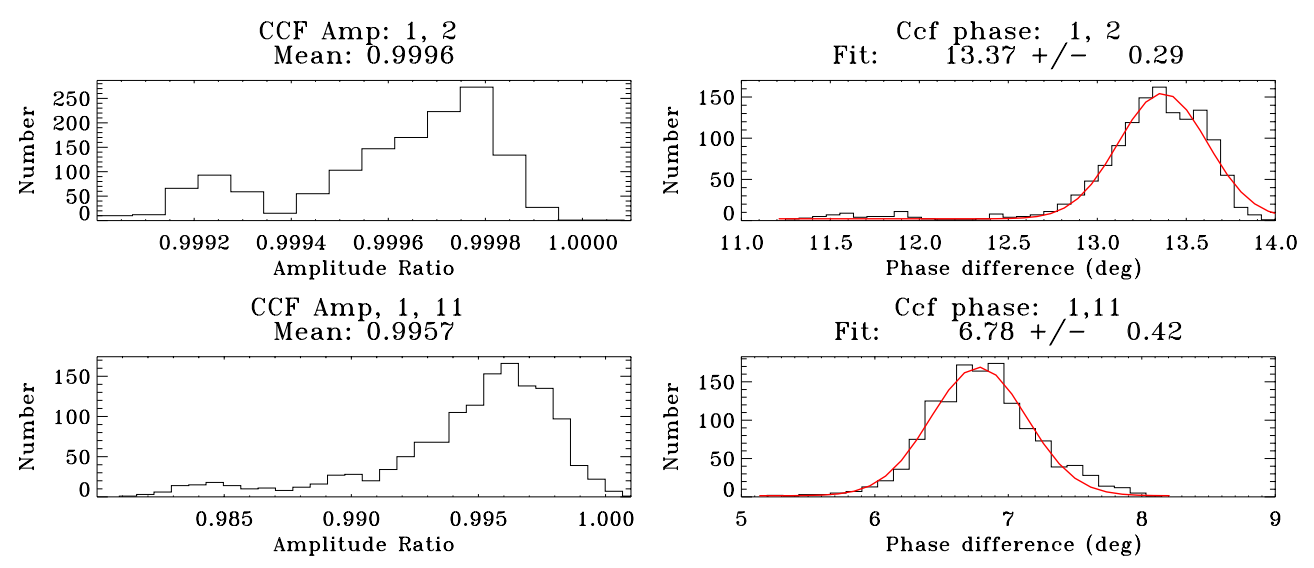

Fig. 1. Histogram of cross-correlation magnitude (left) and phase (right) estimated using receiving channels 1 and 2 (top) and 1 and 11 (bottom).

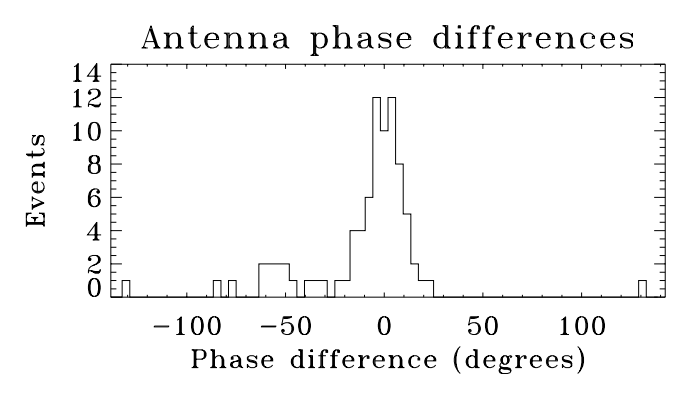

Fig. 2. Histogram of antenna phase differences.

\section{The Buckland Park MF radar}

The Buckland Park MF radar is located $35 \mathrm{~km}$ North of Adelaide $\left(34^{\circ} 38^{\prime} \mathrm{S}, 138^{\circ} 29^{\prime} \mathrm{E}\right)$, and operates at a frequency of $1.98 \mathrm{MHz}$. The antenna array consists of a $1-\mathrm{km}$ diameter array of 89 individually accessible north-south and east-west aligned half-wave dipoles. The radar was upgraded between 1991 and 1995 (e.g. Reid et al., 1995), involving the refurbishment of the entire antenna array and the commissioning of new transmitting and radar data acquisition (RDAS) systems. The antenna array can now be used for transmission, enabling the BPMF to operate as a Doppler radar (e.g. Vandepeer and Reid, 1995). The flexibility of the radar allows one to use it for specialized experimental campaigns. Routine spaced antenna observations are performed outside campaigns and maintenance periods.

The transmitting system consists of three 10-channel solid-state modules, each of which can be used as an individual transmitter. Each transmitter channel consists of a poweramplification (PA) module, a phase control module (PCM), and a transmit-receive (T/R) switch. The PA modules for transmitters 1 and 2 each produce $2.5 \mathrm{~kW}$ nominal power, while those for transmitter 3 each produce $5 \mathrm{~kW}$ nominal power. The maximum total RMS peak envelope power for the system is, therefore, $100 \mathrm{~kW}$. Each transmitter channel is connected to three dipoles of the antenna array, and these dipoles can also be used for reception via the T/R switches. The PCMs allow the phase of the transmitted signal for each channel to be adjusted in $8.5^{\circ}$ increments, allowing the transmitter polar diagram to be steered off-zenith in any direction. The restriction to $8.5^{\circ}$ multiples produces negligible deterioration of the polar diagram in comparison to that obtained using exact phasing. Each transmitter produces Gaussianshaped pulses with half-power full-width (HPFW) $14 \mu \mathrm{s}$, corresponding to a range resolution of $\approx 4 \mathrm{~km}$ HPFW. The duty cycle of each transmitter is approximately $0.2 \%$.

The radar data acquisition system (RDAS) consists of 16 receiving channels, each comprising of a receiver and signal processor. Each channel can be connected to individual dipoles or to groups of three dipoles, including those employed for transmission via the T/R switches. The signal processors use 12-bit digitisation, which is increased to 16-bit upon coherent integration. The receiver bandwidths corresponds to a transmit HPFW of $2 \mathrm{~km}$, which is half the transmit pulse duration. This bandwidth was chosen with the use of narrower transmit pulse widths and pulse coding in mind. Despite the bandwidth being nonoptimal, the combination of the wide beamwidth and narrow transmit beam minimises range smearing. The signal returns can be sampled at a $1-$ or 2-km range resolution.

The radar is controlled by a DOS-based (acquisition) PC which writes each individual raw data acquisition to file, and then transfers the file to a Linux (analysis) PC, where it is queued for analysis. The analysis is part of the "Analysis and Display suite" (ADS), a commercially available package supplied by Atmospheric Radar Systems Pty. Ltd. (ATRAD). The ADS consists of analysis, postanalysis, and display modules. The analysis allows each raw data record to be analysed using a number of different techniques, and to be stored as spectra and covariance functions. The postanalysis allows incoherent averaging of spectra and covariance functions for further analysis, and processing of analysis products, such as hourly averages and the production of "plot files" of the analysed data results which can be sent to remote locations for display on web 
pages via ftp. The latter functionality provides hourly updated latest result plots, as displayed at the web addresses http://www.physics.adelaide.edu.au/atmospheric and http:// www.atrad.com.au/results.html.

\subsection{Receiver and antenna evaluation}

Receiver characteristic differences can produce biased wind estimates when using the full correlation analysis (FCA). This bias is known as the "triangle size effect" (hereafter TSE) (e.g. Golley and Rossiter, 1970; Meek, 1990; Holdsworth, 1999b), whereby the FCA "true" velocity decreases with decreasing antenna spacing. Furthermore, phase differences between antenna systems combined for use by the FCA can also introduce the TSE (e.g. Holdsworth, 1999b). As a result, a number of tests were performed prior to routine analysis implementation, to investigate the contribution of these factors upon the FCA TSE, and for design of an optimal experimental configuration to reduce their effects.

Receiver characteristic differences were determined as described by Golley and Rossiter (1970). A commercially available 8-way splitter was used to split the signal received by a single antenna into multiple receivers. Cross-correlation functions (CCFs) of the resulting signals were calculated. After interpolating across zero-lag to remove the effects of noise correlated between receiver channels (e.g. Briggs, 1984), the zero-lag CCF magnitudes were estimated to measure the statistical similarity of the receiving channels, and the zero-lag CCF phases were estimated to measure the phase difference between channels. Examples of cross-correlation magnitude and phase histograms for two receiver pairs resulting from 200 2-min observations using all ranges with signal-to-noise ratios (SNRs) exceeding $10 \mathrm{~dB}$ are shown in Fig. 1. The mean of the correlation magnitude distribution for channels 1 and 2 is slightly less than unity, indicating slightly different receiver responses. This mean is typical of the values obtained comparing receivers 1-10 (and 11-16), which range from 0.999 to 1.0 . In contrast, the means obtained using channels 1 and 11 are significantly smaller than unity. These means are typical of the values obtained comparing receivers $1-10$ with those from $11-16$, which range from 0.990 to 0.995 . This is due to minor design differences between the two groups of receivers. A similar procedure was performed to compare the characteristics of the $T / R$ switches, yielding correlations in the range of 0.999 to 1.0 . Although a $1 \%$ departure from unity may seem a small and acceptable value, FCA velocity magnitude biases as large as $20 \%$ can occur for some combinations of antenna spacing and pattern scales appropriate for the Buckland Park MF radar. For this reason, it was decided to apply FCA using receivers 1 to 10 only.

Antenna system phase differences may be introduced by the antennas, baluns, or feeder cables. Antenna system phase differences were determined using zero-lag cross-covariance phases (or ZCCPs) obtained from several one-hour runs of 2-min observations using all ranges with SNRs exceeding $10 \mathrm{~dB}$. The implicit assumption used is that the mean an- gle of arrival (MAOA) for each record should be distributed about the zenith (e.g. Kudeki et al., 1990). Profiles of the ZCCPs often exhibited significant offsets above $86 \mathrm{~km}$, which can be attributed to the ionosphere. This behavior is consistently observed for the BPMF radar (K. J. Berkefeld, private communication). Similar offsets are observed for the Urbana MF radar by Thorsen et al. (1997). As a result, ZCCPs below $86 \mathrm{~km}$ were used to estimate the antenna phase differences. The resulting mean ZCCP measure the phase differences introduced by the antenna system and the receiver. The receiver phase differences determined from the receiver characteristic tests described above are then subtracted to yield the phase differences introduced by the antenna system alone. The resulting distribution of antenna phase differences with respect to an arbitrarily selected "good" antenna for each dipole orientation are shown in Fig. 2. The majority of the phase differences are less than $20^{\circ}$. Time Domain Reflectometry (TDR) measurements of antennas producing phase differences exceeding $20^{\circ}$ often indicated the presence of water in the air-cored coaxial cable. This behavior produces the assymetry evident in the phase histogram as water acts to increase the electrical length of the cable, thereby producing large negative phase differences when compared with good antennas. Maintenance procedures are ongoing to improve such antennas and any other antenna related problems that may arise (e.g. Grant, 2004).

\subsection{Antenna configuration and transmitter polar diagrams}

Simulations of the transmitter polar diagram obtained using the entire BPMF antenna array for transmission suggest that randomly distributed phase differences less than $20^{\circ}$ produces negligible degradation of the polar diagram (e.g. Vandepeer, 1993). However, phase differences of this order can produce FCA TSE biases when groups of three antennas are combined for reception (e.g. Holdsworth, 1999b). As a result, antennas with phase differences less than $20^{\circ}$ have been preferentially selected for transmission, while antennas with phase differences less than $10^{\circ}$ have been preferentially selected for reception.

The antenna configuration has been modified throughout the observations as necessitated by antenna failures, reavailability of corrected antennas, transmitter availability, and requirements to test different antenna configurations for particular analyses. Transmission is performed using the northsouth aligned antennas, producing a linearly polarized signal. The number of transmit antennas and the total transmitter power for different observation periods are shown in Table 1. Except for a four month period in 1999, either 30 or 60 antennas have been used for transmission. The antenna configuration employed for the routine observations between 16 July 1997 and 4 January 1998 is shown in Fig. 3. The transmit polar diagrams obtained using the first 30 antennas (transmit modules 1 to 10) and all 60 antennas (transmit modules 1 to 20) of this configuration are shown in Fig. 4. These polar diagrams are typical of those produced using 30 and 60 antennas throughout the observations, producing half-power 


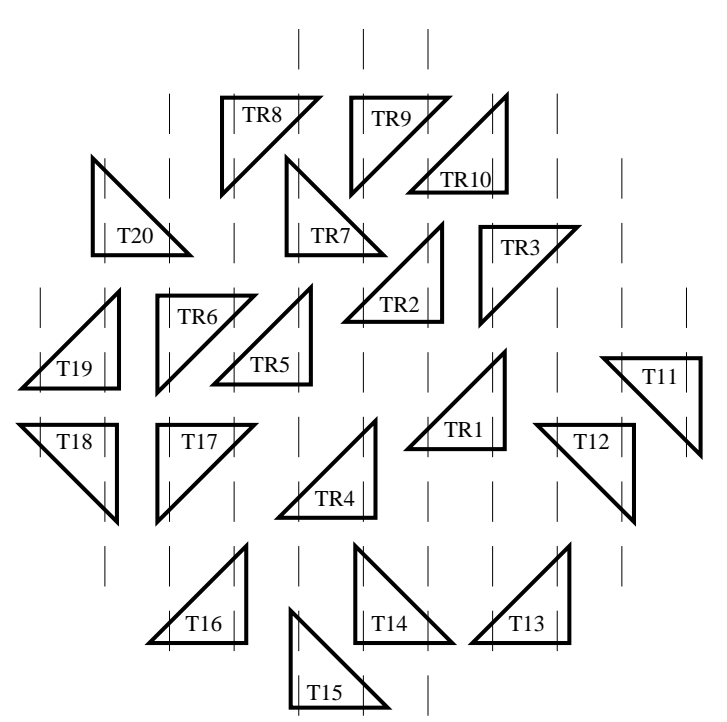

North/South Array

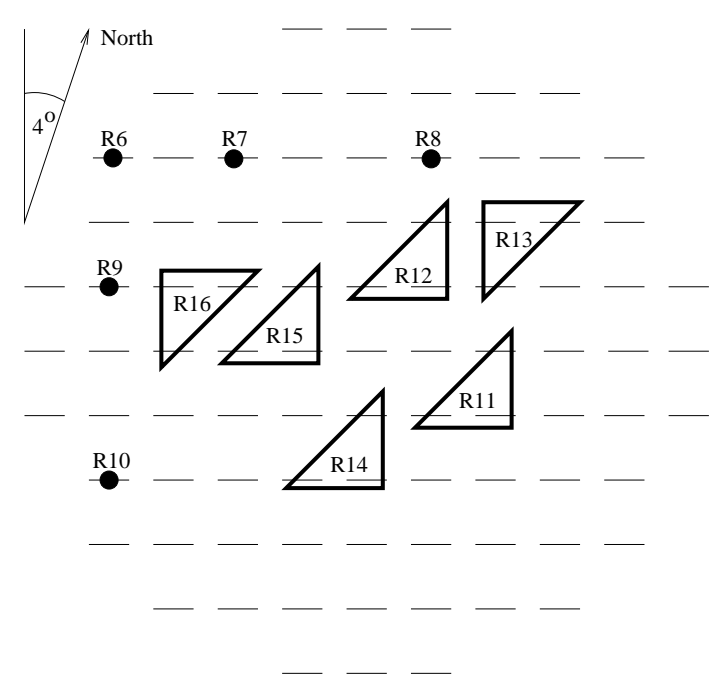

East/West array

Fig. 3. Antenna configuration employed for initial routine observations using the Buckland Park MF radar. Each vertical line on the north/south array (left) represents a single north-south aligned antenna, while each horizontal line on the east/west array (right) represents a single east/west aligned antenna. The triangles denote the antennas used for observations, and the appropriate transmit channel. Antenna groups denoted $\mathrm{TR}_{i}$ and $\mathrm{T}_{i}$ were connected to transmitter $i$. Antenna groups denoted $\mathrm{TR}_{i}$ were connected to receiver $i$ via $\mathrm{T} / \mathrm{R}$ switch $i$. Antenna groups denoted $\mathrm{R}_{i}$ were connected directly to receiver $i$. The filled circles denoted $\mathrm{R}_{i}$ were connected to receiver $i$ for meteor observations from March 2000.
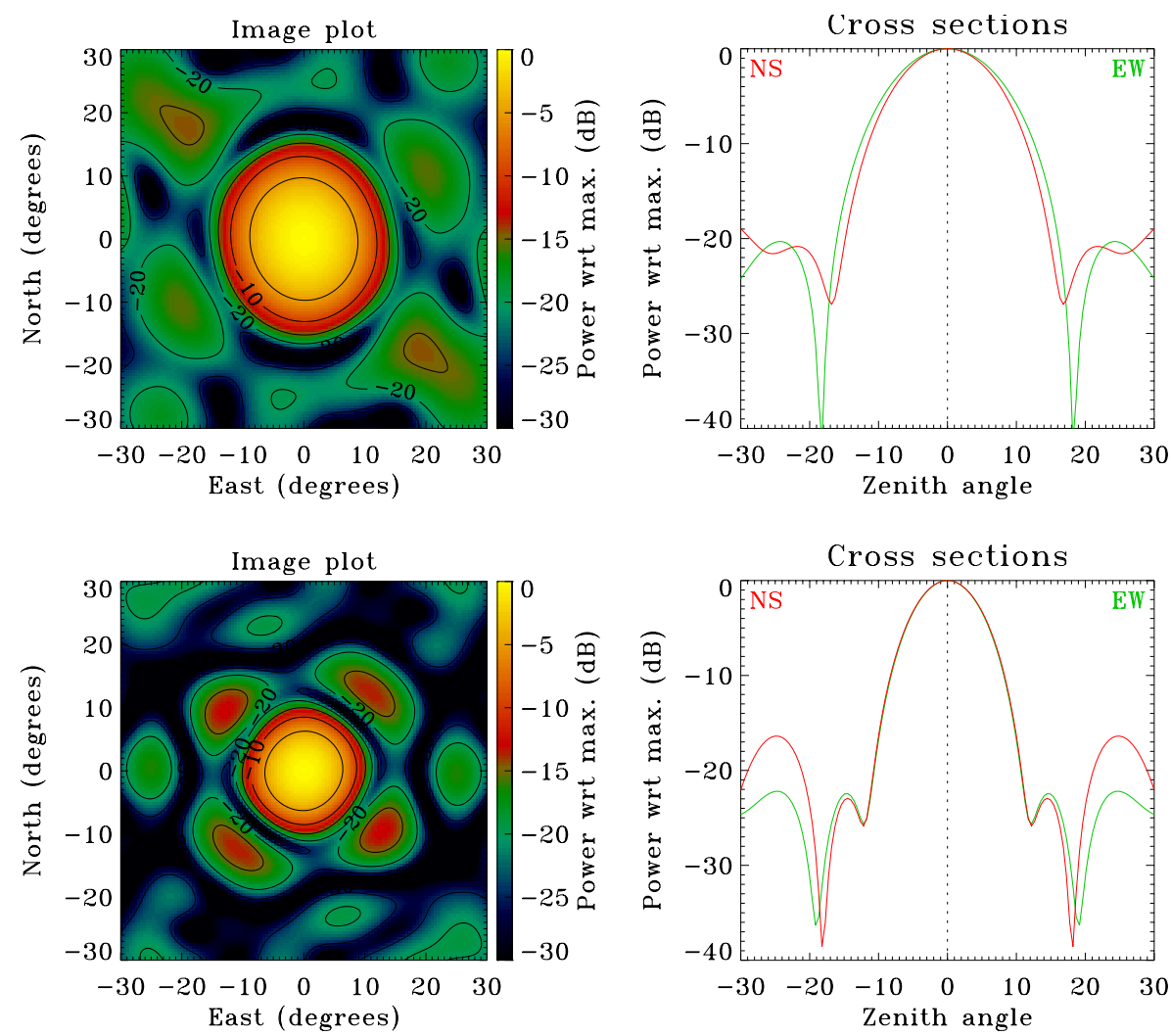

Fig. 4. Polar diagrams employed for routine observations using the Buckland Park MF radar. The top plots show image (left) and crosssections (right) plots obtained using channels 1 to 10 . The bottom plots show the corresponding plots using channels 1 to 20 . 
Table 1. Observation periods for routine Spaced Antenna (SA) analysis for the Buckland Park MF radar.

\begin{tabular}{lccc}
\hline \multicolumn{1}{c}{ Observation period } & Number of antennas & Transmit HPHW (deg) & Peak Power (kw) \\
\hline $7 / 5 / 1996$ to $16 / 7 / 1997$ & 30 & 8.4 & 25 \\
$16 / 7 / 1997$ to $4 / 1 / 1998$ & 60 & 5.7 & 75 \\
$19 / 3 / 1998$ to $23 / 4 / 1998$ & 30 & 8.4 & 25 \\
$24 / 3 / 1998$ to $29 / 6 / 1998$ & 60 & 5.7 & 50 \\
$2 / 3 / 1999$ to $31 / 7 / 1999$ & 75 & 5.0 & 82.5 \\
$1 / 8 / 1999$ to present & 60 & 5.7 & 50 \\
\hline
\end{tabular}

half-widths of $8.4^{\circ}$ and $5.7^{\circ}$, respectively. Apart from a small degree of asymmetry, the polar diagrams otherwise show no undesirable features, despite the irregular antenna grid employed. The maximum sidelobe levels are $13 \mathrm{~dB}$ below the peak power level.

Receivers 1 to 10 were initially connected to T/R switch outputs 1 to 10 , and, therefore, to the north-south antenna groups 1 to 10 . Receivers 11 to 16 were connected to the east-west aligned antennas corresponding to north-south antenna groups 1 to 6 . Receiver 16 was connected to T/R switch 1 via an attenuator from 19 March 1998 onwards, to facilitate observations of descending E-region layers (e.g. Holdsworth et al., 2001), which generally saturate the receivers when used with the optimal receiver gain setting used for routine observations. Receivers 6 to 10 were connected directly to 5 east-west antennas from 14 September 2000 onwards, to provide an interferometer for meteor observations.

\subsection{Complex gain difference estimation and correction}

Compensation for receiver channel complex gain differences is critical for accurate interferometric analyses, such as TDI, HDI, IDI and meteor analyses, and for accurate decomposition of the O- and E-mode signals for DAE analysis. Although the BPMF radar is capable of automated receiver calibration (e.g. Reid et al., 1995), these measurements do not account for phase delays through the T/R switches, feeder cables and antennas. The receiver channel complex gain differences are, therefore, estimated using a receiver channel calibration (RCC) procedure. This procedure is typically performed monthly, using at least one day of daytime raw data archived by the routine analysis. Daytime data is preferred due to its higher SNR and lower interference level. The RCC is only applied using data with SNRs exceeding $10 \mathrm{~dB}$, and is applied as follows.

The receiver channel amplitudes $A_{i}$ are estimated by calculating the zero-lag auto-covariance function magnitudes after interpolating over zero-lag to remove the effects of noise. The mean receiver channel amplitude ratios with respect to a selected reference receiver $j$ are then estimated using $\overline{A_{i j}}=\overline{A_{j} / A_{i}}$. The receiver channel phase differences $\theta_{i j}$ are estimated using the ZCCP with respect to a reference receiver channel $j$ after interpolating over zero-lag to remove the effects of noise correlated between receiver chan- nels. The mean receiver channel phase differences $\overline{\theta_{i j}}$ are then estimated using the first moments from a Gaussian fitting, which is preferred to the mean as it is less influenced if the distribution is skewed or has significant tails. As described in Sect. 2.1, the implicit assumption used in this step of the RCC is that the MAOA over the observation period is on zenith. This is thought to be a valid assumption for the 8-h (or more) data sets used for the RCC. The complex gain correction used for multiplying each receiver channel $i$ to correct for complex gain differences is then $\overline{A_{i j}} \exp -i \overline{\theta_{i j}}$. Monthly estimated amplitude ratios and phase differences typically showed consistency to within the RMS values of the distributions on a month to month basis. The largest departures observed are usually associated with antenna system failures within a group of three antennas, and are typically of the order of $10 \%$ for amplitude ratios and $20^{\circ}$ for phase differences.

The RCC procedure is first performed for receivers 1 to 10 , yielding the amplitude ratios and phase differences with respect to channel 1. For the same reasons described in Sect. 2.1, only phase differences below $86 \mathrm{~km}$ were used to estimate the receiver channel phase differences for receivers 1 to 10 . The amplitude ratios were calculated using data from all ranges below $90 \mathrm{~km}$. This limit was selected as receiver saturation can occur above $90 \mathrm{~km}$, which can have significant effects on the estimated amplitude ratios.

The histogram procedures were then repeated for the crossed dipole receiver pairs $1-11,2-12,3-13,4-14,5-$ 15 and 6-16, using daytime data from ranges between 80 and $90 \mathrm{~km}$. The upper limit was selected due to the possibility of receiver saturation, which can have significant effects upon the estimated phase differences. The lower limit was selected as the $\mathrm{O}$-mode signal above $80 \mathrm{~km}$ is significantly stronger than the E-mode signal during the day. The resulting phase difference between each pair of cross-dipoles is therefore $\approx 90^{\circ}$, allowing for the determination of the phase difference between the two crossed dipoles. This allows for the amplitude and phase differences to be combined into a gain correction with respect to receiver 1 , thereby providing the same complex gain reference for all receiver channels used in the DAE experiment. The range limiting procedure is essentially the same as suggested by Von Biel (1977) for the complex gain calibration for polarimetric observations. 

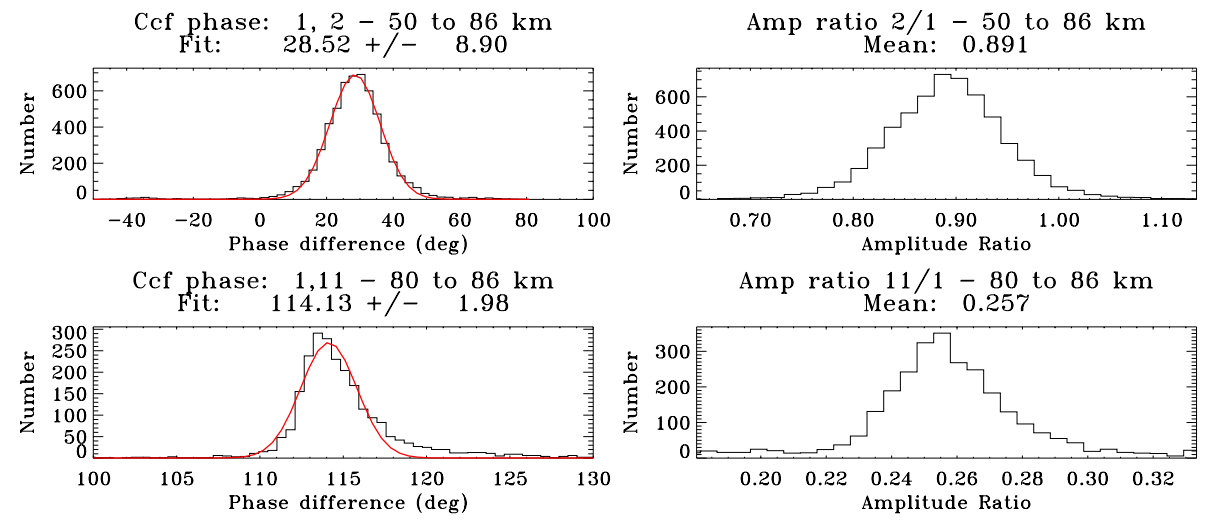

Fig. 5. Examples of histograms of phase differences (left) and amplitude ratios (right) obtained by applying the RCC to daytime data from between 4 and 9 April 2000. The red lines on the phase differences plots show the result of applying a Gaussian fit.

Table 2. Experimental parameters used for routine Spaced Antenna (SA) analysis for the Buckland Park MF radar.

\begin{tabular}{lcc}
\hline \multicolumn{1}{c}{ Parameter } & Day Value & Night Value \\
\hline Start range, km & 50 & 70 \\
Sampling range resolution, km & 2 & 2 \\
Number of range & 25 & 15 \\
Polarization & linear & linear \\
PRF, Hz & 100 & 20 \\
Coherent integrations & 40 & 8 \\
Effective sampling time, s & 0.4 & 0.4 \\
Number of samples & 280 & 280 \\
Record length, s & 112 & 112 \\
\hline
\end{tabular}

An example of selected histograms of amplitude ratios and phase differences obtained on applying the RCC for daytime data between the 4 and 9 April 2000 is shown in Fig. 5. For routine observations from 14 September 2000 incorporating the meteor interferometer, the RCC for channels 6 to 10 was applied as a separate subset, with channel 6 used as the reference channel.

\subsection{Routine observations}

Routine observations commenced 7 May 1996, using the parameters shown in Table 2. Data acquisition is performed for $112 \mathrm{~s}$, with the following $8 \mathrm{~s}$ used for transfer of the $\approx 1.5 \mathrm{Mb}$ raw data files between the acquisition and analysis PCs. The only modification to these parameters has been the extension of the day (night) height range maximum to 158 (178) km from 19 March 1998, to allow for studies of descending total reflection layers. It is important to emphasize that each of the analyses described in Sects. 3 to 9 can be applied to each individual 2-min raw data set. Receiver calibration is applied prior to analysis. Data above $98 \mathrm{~km}$ is only analysed by the DBS analysis configured for the attenuated signal of receiver 16 , and the meteor analysis. All routine observations outside specialised Doppler campaigns use vertical beam transmission.

\section{Full correlation analysis}

Full correlation analysis (FCA) (e.g. Briggs, 1984) has been applied throughout the observations, providing estimates of the dynamics and the spatial and temporal properties of the radiowave scatterers. The FCA has used antennas with similar spacings to those connected to receivers 1, 2 and 3 (hereafter "FCA-small") shown in Fig. 3. On 2 March 1997 a second FCA analysis was implemented, using antennas with larger spacing, such as those connected to receivers 1, 6 and 9 (hereafter "FCA-large"). Preliminary FCA results are presented by Holdsworth and Reid (1997) and Holdsworth et al. (2001). Further FCA results are presented in the accompanying paper Holdsworth and Reid (2004).

The initial selection of a small spacing may be considered unusual since FCA is known to be affected by the TSE. The motivation for using the smaller spacing was threefold. First, simulations suggest smaller spacings produce smaller measurement errors (e.g. Holdsworth, 1999b). This can be attributed to smaller spacings using cross-correlation functions with higher correlations, and hence smaller correlation parameter errors. Second, smaller spacing reduces the occurrence of cases where the antenna spacing exceeds the pattern scale. This results in low cross-correlation between the signals at each antenna, increasing the occurrence of spurious velocity estimates due to the use of correlation parameters with large errors, or correlation parameters estimated using incorrectly identified cross-correlation maxima. The FCA therefore applies criteria (e.g. Briggs, 1984) to reject low cross-correlation maxima, large delay-times to crosscorrelation maxima, and large normalised time-discrepancies (NTDs, the ratio of the sum of the delay times and sum of the absolute delay times), since NTD should be zero. Third, and perhaps most importantly, we felt confident that we had eliminated (or at worst minimised) all known TSE sources. It was later realised there were two further TSE sources that we 
Table 3. Mean and standard deviation of daily relative magnitude minima and maxima, correlation, maximum measurement errors, and acceptance rates for FCA-large relative to FCA-small from 2 March 1997 to 19 September 2003.

\begin{tabular}{lcc}
\hline Parameter & Zonal component & Merid. component \\
\hline Relative magnitude minimum & $1.20 \pm 0.07$ & $1.18 \pm 0.07$ \\
Relative magnitude maximum & $1.22 \pm 0.02$ & $1.22 \pm 0.08$ \\
Correlation & $0.91 \pm 0.02$ & $0.88 \pm 0.03$ \\
FCA-small measurement error, $\mathrm{ms}^{-1}$ & $10.0 \pm 1.1$ & $9.9 \pm 1.1$ \\
FCA-large measurement error, ms & \\
FCA-small acceptance rate, $\%$ & $10.5 \pm 1.2$ & $10.5 \pm 1.3$ \\
FCA-large acceptance rate, \% & 66.3 & 66.3 \\
\hline
\end{tabular}

Table 4. Mean and standard deviation of daily relative magnitude minima and maxima for "large-FCA" and "O-mode large-FCA" from 1 January 2001 to 25 July 2001.

\begin{tabular}{lcc}
\hline Parameter & Zonal component & Merid. component \\
\hline Relative magnitude minimum & $0.96 \pm 0.02$ & $0.96 \pm 0.02$ \\
Relative magnitude maximum & $0.96 \pm 0.02$ & $1.00 \pm 0.02$ \\
\hline
\end{tabular}

could not eliminate: different configurations of combined antennas, and nonsimultaneous range sampling. The former is evident in Fig. 1, where antenna 3 has a different orientation from antennas 1 and 2. Selecting groups of antennas with identical orientations is unfeasible, given the dual requirements of avoiding the use of bad antennas and maintaining satisfactory polar diagrams. The latter results from the different feeder cable lengths used in the antenna array (e.g.

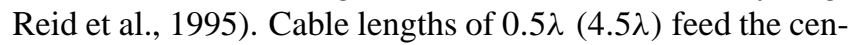
ter (outer) antennas. The range gates sampled by the outer antennas are therefore $681 \mathrm{~m}$ lower than the center antennas. This represents a significant fraction (0.17) of the nominal transmit pulse half-power full-width $(4 \mathrm{~km})$ - although we note the different cable lengths will act to smear the transmitted pulse. Radar backscatter modeling (e.g. Holdsworth and Reid, 1995) of these effects suggested that the smaller spacing should produce an underestimation of at most $10 \%$.

The FCA results have been used to investigate the TSE using the statistical comparison technique of Hocking et al. (2001), and the extension described by Holdsworth and Reid (2004). The daily mean relative magnitudes, correlations, "maximum" measurement errors and acceptance rates are shown in Table 3. The relative magnitudes indicate that FCA-small velocities are $\approx 20 \%$ smaller than FCA-large, indicating TSE. The FCA-small underestimation exceeds the expected value of $10 \%$, suggesting a further unaccountable TSE source. The correlations are around 0.9, suggesting excellent agreement. The estimated measurement errors for FCA-small are 5\% smaller than those for FCA-large, which may only reflect the fact that the relative magnitudes are smaller. The FCA-large acceptance rate is $11 \%$ lower than FCA-small. The lower FCA-large acceptance rate results from large NTD and low cross-correlation maxima at heights above $90 \mathrm{~km}$, justifying the second motivation for our initial selection of the smaller spacing for FCA analysis.

Another source of TSE suggested by Holdsworth (1999b) is complex gain errors in the decomposition of the signals received by crossed dipoles into O- and E-mode circular polarisation. Between 1 May 2001 and 25 July 2001 the FCA analysis was also applied to the O- and E-mode signals determined for the antennas used for FCA-large, as calculated for use in DAE analysis. The relative magnitude for Omode winds in comparison with FCA-large are shown in Table 4 , suggesting that the winds estimated using circularly polarised signals are slightly underestimated, which is consistent with the suggestions of Holdsworth (1999b). The BPMF routine analysis $\mathrm{O}-$ and $\mathrm{E}$-mode signals are determined post-receiver, which is not the case for most MF/HF radar systems. Regardless of the means by which circularly polarised signals are determined, there will be potential sources of complex gain errors. The results of Table 4 suggest less biased velocity estimates may occur on applying FCA using linearly polarised antennas, as is the case for the BPMF routine analysis.

The annual variation of the FCA true velocity components, aspect sensitivity parameters $\left(\theta_{s}\right)$, and turbulent velocities obtained using data from 07 July 1996 to 19 September 2003 are shown in Fig. 6. These plots represent fortnightly averages superposed into a single year: the 2-min data are averaged to produce hourly averages, which are then used to produce daily averages, which are then used to produce fortnightly averages. FCA-small results are used until 2 March 1997, and FCA-large results thereafter. The averaging method used gives each hourly average equal weight in the daily average, and each daily average equal weight in the fortnightly average. This is preferred to a fortnightly 

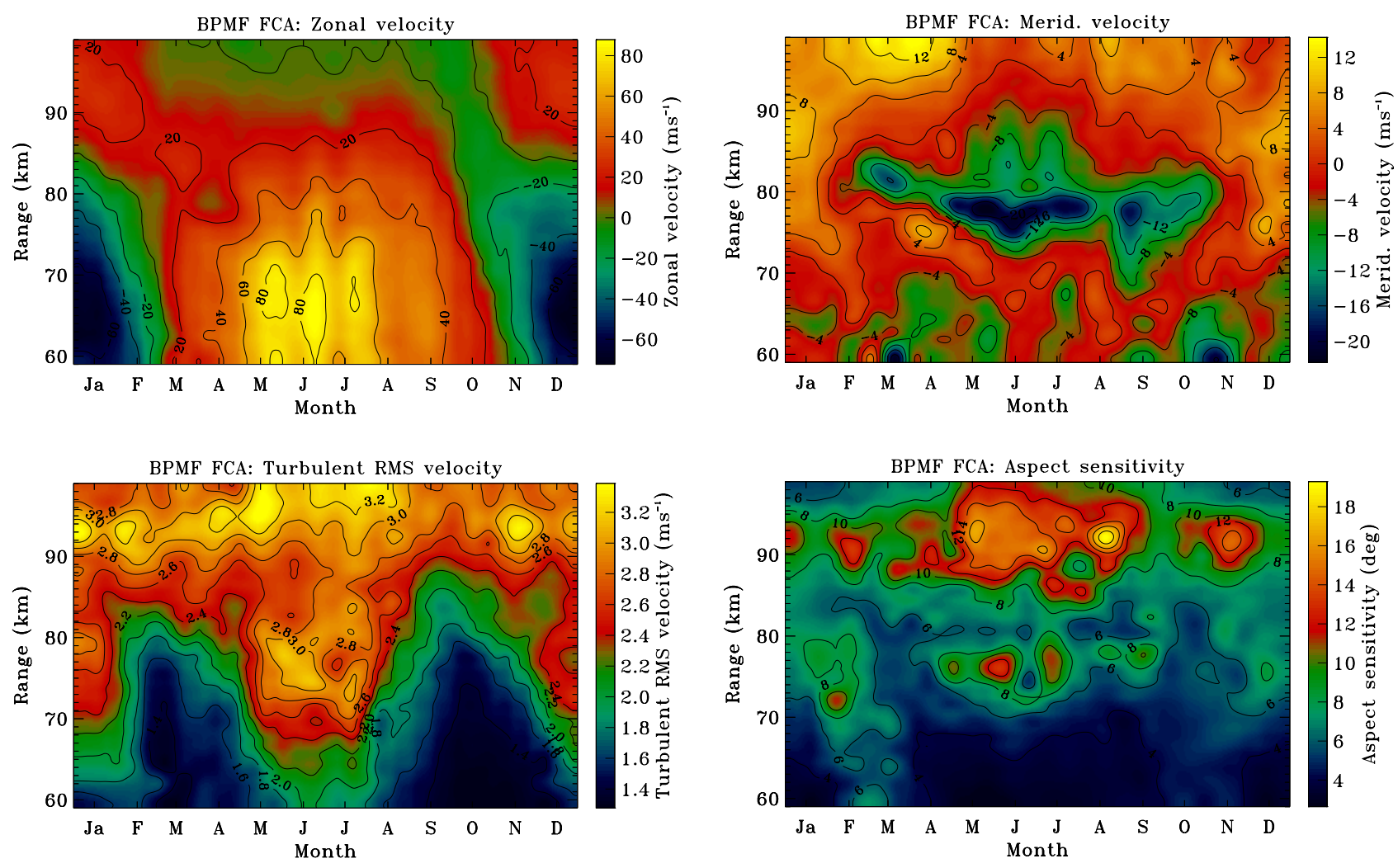

Fig. 6. Superposed annual variation of BPMF FCA parameters zonal velocity (top left), meridional velocity (top right), turbulent RMS velocity (bottom left), and aspect sensitivity (bottom right).

average of the 2-min data, which would bias the averages towards the values obtained when the acceptance rates are largest. The zonal velocities above $84 \mathrm{~km}$ are predominantly eastwards except between mid-September to mid-November. The eastward/westward reversal height descends from $94 \mathrm{~km}$ in mid-September to $60 \mathrm{~km}$ in mid-November, a descent of approximately $0.56 \mathrm{~km}$ per day. Below $84 \mathrm{~km}$ the zonal velocities show the eastward winter jet and westward summer jet typically observed at mid-latitude stations (e.g. Manson et al., 1991). The winter jet shows greater variability than the summer jet, with peaks of $85 \mathrm{~m} \mathrm{~s}^{-1}$ at $68 \mathrm{~km}$ in late May, June and July, compared to a single summer jet peak of $-68 \mathrm{~m} \mathrm{~s}^{-1}$. The winter peak times vary from year to year, but there are always two or three peaks each winter. The greater variability of the winter jet may be indicative of stratospheric warming (e.g. Manson et al., 1991), or possibly the failure of our averaging procedures to properly filter out planetary waves (e.g. Nakamura et al., 1996). The summer reversal height is around 84 to $85 \mathrm{~km}$, consistent with previous mid-latitude Southern Hemisphere observations (e.g. Manson et al., 1987). The meridional velocities are predominantly northward above $70 \mathrm{~km}$, with the exception of a strong southward peak extending from $82 \mathrm{~km}$ in March, to $77 \mathrm{~km}$ in June, and back up to $80 \mathrm{~km}$ in October, and weaker southward flows between the peak and $90 \mathrm{~km}$ in winter. Below $70 \mathrm{~km}$ the velocities are predominantly southward, and show considerable variability. Turbulent velocities are estimated as described by Briggs (1980), and increase with height. Solstice maxima and equinoctal minima are observed below $80 \mathrm{~km}$. There is evidence of a maximum extending from $93 \mathrm{~km}$ in summer to above $98 \mathrm{~km}$ in winter. We attribute this to leakage from E-region total reflection, since harmonic analysis of BPMF turbulent velocities has been shown to yield turbulent velocities whose maxima peak at midday (e.g. Holdsworth et al., 2001). The aspect sensitivity parameter $\theta_{s}$ is estimated using the "spatial correlation technique" of Lesicar and Hocking (1992a), and increases with height, indicating less aspect sensitive scatter. The exceptions are winter and summer maxima at $76 \mathrm{~km}$, and weak maxima at all heights below $74 \mathrm{~km}$ in early March and October. There is a weak minimum associated with the strong southward meridional velocity peak.

The above results are in qualitative agreement with the zonal and meridional velocities of Lesicar (1993) and the aspect sensitivities of Lesicar et al. (1992b), who presented a similar climatology using data from the previous BPMF radar, which used transmission on a separate 4-antenna dipole array with a large beam width, and an 8-bit data acquisition system. The main difference is that the velocities presented in the current results are approximately $30 \%$ larger, and the aspect sensitivities are approximately 50\% smaller. This is consistent with the hypothesis that the new 

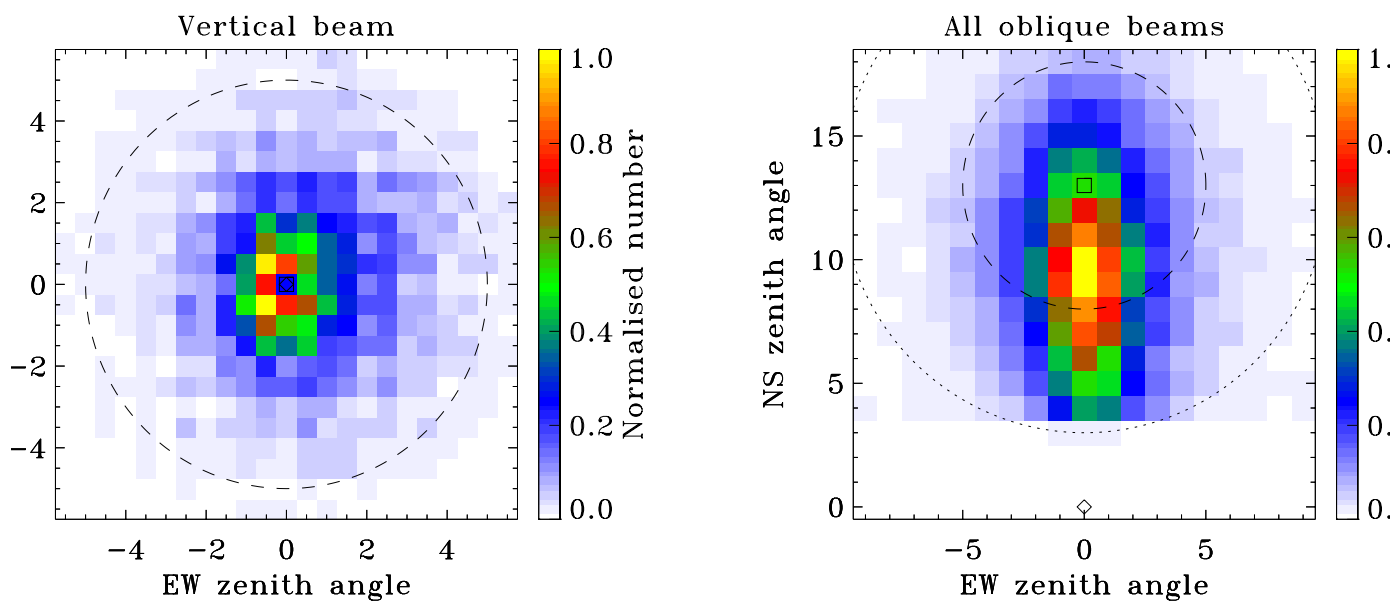

Fig. 7. Histograms of effective beam positions estimated during the 5-beam Doppler campaign July 1998. The left plot shows vertical beam, while the right plot shows the combined results from the north, east, south and west beam directions, with the east, south and west beams results rotated to the north. The squares indicates the beam direction, and the diamonds indicates the zenith.

BPMF radar is less susceptible to TSE biases. The transmit beamwidths are considerably smaller than those usually used for most $\mathrm{MF} / \mathrm{HF}$ radar observations $\left(20^{\circ}-40^{\circ}\right)$ (e.g. Vincent and Lesicar, 1991). FCA measurement errors have been shown to increase with decreasing beamwidths (e.g. Røyrvik, 1983; Kawano et al., 2002), as the resulting increase in the ground diffraction pattern scale increases the errors in the correlation parameters used in the analysis. However, the measurement errors in the correlation parameters decrease with increasing SNR. Since increasing the flux of power directed vertically (from which the strongest returns emanate) increases SNR, it is expected that any increase in measurement errors due to pattern scale increases are more than compensated for. The increased SNR also extends the lowest observation range - Holdsworth and Reid (1997) show regular winter daylight velocity estimates down to $52 \mathrm{~km}$, considerably lower than that achieved using previous BPMF radar systems.

\section{Differential absorption experiment}

The Differential absorption experiment (DAE) (e.g. Gardner and Pawsey, 1953) was implemented in August 1996 for the estimation of electron densities and collision frequencies. Receiver outputs 1 to 5 are combined with receiver outputs 11 to 15 to form five pairs of ordinary (O-mode) and extraordinary (E-mode) signals. The five O-mode signals are combined to produce the O-mode signal used for analysis, and the five E-mode signals similarly are combined to produce the E-mode signal. Detailed information regarding the BPMF DAE analysis and electron density estimates are presented by Holdsworth et al. (2002), while collision frequency estimates are presented by Vuthaluru et al. (2002).
Table 5. Mean and standard deviation of daily relative magnitude minima and maxima, correlation, maximum measurement errors, and acceptance rates for SCA relative to FCA-small from 18 October 1996 to 1 May 1997.

\begin{tabular}{lcc}
\hline Parameter & Zonal component & Merid. component \\
\hline Relative magnitude minimum & $1.24 \pm 0.08$ & $1.30 \pm 0.05$ \\
Relative magnitude maximum & $1.32 \pm 0.03$ & $1.34 \pm 0.04$ \\
Correlation & $0.95 \pm 0.01$ & $0.91 \pm 0.03$ \\
SCA measurement error, $\mathrm{ms}^{-1}$ & $10.7 \pm 0.8$ & $10.8 \pm 0.7$ \\
FCA measurement error, $\mathrm{ms}^{-1}$ & $9.7 \pm 0.4$ & $9.7 \pm 0.5$ \\
SCA acceptance rate, $\%$ & 30.7 & 30.7 \\
FCA acceptance rate, $\%$ & 60.3 & 60.3 \\
\hline
\end{tabular}

\section{Spatial correlation analysis}

The revised spatial correlation analysis (SCA) (e.g. Holdsworth, 1999a) was implemented on 18 October 1996. The SCA was applied to the square of receiving channels 1 , 2, 4 and 5 shown in Fig. 3. Antenna degradation throughout the observations made maintaining the square configuration difficult, and SCA was discontinued on 1 May 1997. The major motivation for investigating SCA was that previous implementation illustrated that the velocities compared well with FCA velocities estimated for large antenna spacings (e.g. Golley and Rossiter, 1970), suggesting a less biased velocity estimate than FCA, which is subject to the TSE. A detailed examination of the performance of the SCA is considered beyond the scope of this paper, although some aspects of the analysis performance are discussed below.

Table 5 shows the results of applying the aforementioned statistical comparison technique to the SCA and FCA-small velocities estimated from 18 October 1996 to 1 May 1997. The relative magnitude maxima suggest the SCA velocities are approximately $30 \%$ larger than the FCA-small veloci- 
Table 6. Mean and standard deviation of daily relative magnitude minima and maxima for HDI relative to DBS from 18 June 1998 to 29 June 1998.

\begin{tabular}{lcc}
\hline Parameter & Zonal component & Merid. component \\
\hline Relative magnitude minimum & $1.19 \pm 0.06$ & $1.36 \pm 0.04$ \\
Relative magnitude maximum & $1.52 \pm 0.01$ & $1.38 \pm 0.06$ \\
\hline
\end{tabular}

Table 7. Mean and standard deviation of daily relative magnitude minima and maxima, correlation, maximum measurement errors, and acceptance rates for HDI relative to FCA-large from 18 June 1998 to 29 June 1998.

\begin{tabular}{lcc}
\hline Parameter & Zonal component & Merid. component \\
\hline Relative magnitude minimum & $1.01 \pm 0.07$ & $1.11 \pm 0.03$ \\
Relative magnitude maximum & $1.06 \pm 0.09$ & $1.17 \pm 0.05$ \\
Correlation & $0.85 \pm 0.02$ & $0.78 \pm 0.02$ \\
HDI measurement error, $\mathrm{ms}^{-1}$ & $15.2 \pm 1.8$ & $16.0 \pm 1.8$ \\
FCA measurement error, $\mathrm{ms}^{-1}$ & $15.8 \pm 2.0$ & $15.8 \pm 1.7$ \\
HDI acceptance rate, $\%$ & 69.1 & 69.1 \\
FCA acceptance rate, $\%$ & 78.5 & 78.5 \\
\hline
\end{tabular}

ties, which, as described above, are underestimated due to the TSE. The correlations exceed 0.9, suggesting excellent agreement. The estimated measurement errors for the FCAsmall are smaller than those for SCA. However, as per the FCA comparisons in Sect. 3, we cannot conclusively determine the actual relative measurement errors, since the estimated relative measurement errors are influenced by the relative magnitudes. The acceptance rate for the SCA is approximately half that of FCA-small. The majority of extra rejections for the SCA are associated with a criterion requiring the cross-correlations for antenna pairs with the same orientation (e.g. 14 and 25 in Fig. 3) agree to within 20\% (e.g. Holdsworth, 1999a). This represents a test for spatial homogeneity of the ground diffraction pattern, which is necessary for successful SCA velocity estimation. Investigation of both MF/HF and tropospheric VHF SCA analysed data shows this criterion occurs most frequently for either isotropic scatter (i.e. ground diffraction scales smaller than the antenna spacing) or specular scatter. It results in $23 \%$ of the rejections above $90 \mathrm{~km}$ for the BPMF data. The high occurrence may be exacerbated by the effects of unequal cable lengths, which can reduce cross-correlations as described in Sect. 3. Further work is intended to loosen this criteria without producing spurious velocity estimates, and to evaluate the implications of the apparent spatial inhomogeneity this criterion suggests upon the FCA.

For completeness we briefly mention that the SCA analysis has also been applied using the Wakkanai MF radar (e.g. Hocke and Igarashi, 1997). This analysis differs from the BPMF analysis only in the application to antennas arranged in a "Y" configuration. Application of the statistical comparison technique to this data yields correlations, measurement errors and acceptance rates show similarity to the values shown in Table 4 . The relative ratios of the SCA to FCA velocities are approximately 1.05, suggesting that the Wakkanai FCA velocities are less biased than the BPMF FCA-large velocities. This is most probably due to the Wakkanai MF radar using a smaller transmit array than the BPMF radar, such that the receive antennas are a better match to the pattern scale, and the FCA is therefore less influenced by TSE biases.

\section{Beam steering}

The Doppler beam steering (DBS) and hybrid Doppler interferometry (HDI) techniques were implemented in April 1997. DBS is applied by combining all receiving channels to form a receive beam in the same direction as the transmit beam and applying standard Doppler analysis (e.g. Woodman and Guillen, 1974), to estimate radial velocity, spectral width, SNR and power. HDI uses post-statistics steering (PSS) (e.g. Kudeki and Woodman, 1990), to estimate the "effective beam position" (EBP), allowing correction for (and estimation of) aspect sensitivity, which reduces the mean angular position of backscatter for off-zenith beam directions (e.g. Röttger, 1981). PSS is used to form a receive beam which is steered through a grid of beam directions. The power for each beam direction is determined, and the EBP is found by applying a 2-D Gaussian to estimate the direction of maximum power. The receive beam is then resteered in the direction of the EBP, and the standard DBS parameters are estimated. The use of PSS allows the EBP to be determined more accurately (and to lower SNRs) than using the MAOA, since it uses combined receiver outputs, while the MAOA is estimated by cross-correlating single receiver outputs. The term "hybrid Doppler interferometry" is used as the technique is a hybrid of Doppler and interferometric techniques, and is used in preference to "time domain interferometry" (TDI) (e.g. Vandepeer and Reid, 1995), since the analysis can be applied in the time or frequency domain. For BPMF observations the technique is applied in the timedomain, since spectra obtained for mesospheric and lower thermospheric MF/HF radar observations are often irregular and non-Gaussian. Although HDI and DBS analyses are intended for off-vertical transmission, the vertical beam used throughout routine observations allows for HDI estimated MAOAs and radial velocities to be used for TDI, allowing dynamics estimates for durations ranging from hours (e.g. Berkefeld, 1994) to months (e.g. Thorsen et al., 1997).

HDI and DBS analyses were only intermittently applied throughout the routine observations until 19 March 1998, when DBS analysis was configured for application to receiver 16 for investigations of E-region descending layers. However, both analyses have been applied during several 5beam Doppler campaigns for momentum flux estimation using the dual-beam technique (e.g. Vincent and Reid, 1983). The EBPs estimated for all four oblique from 18 to 29 June 1998 are shown in Fig. 7. The most common EBP is 9.5', 
yielding twice as many estimates as at the actual beam position of $13^{\circ}$. Table 6 shows the statistical comparison of the DBS and HDI techniques for 18 to 29 June 1998, while Table 7 shows the comparison for the HDI and FCA-large velocities. The HDI and FCA velocities show good agreement, while the standard Doppler velocities are underestimated due to the EBP being closer to zenith. A more extensive evaluation of the 5-beam Doppler results will be presented in a future paper.

\section{Meteor analysis}

Following the successful BPMF meteor observations of Tsutsumi et al. (1999), the Atrad VHF meteor analysis software (e.g. Holdsworth et al., 2004) was modified for BPMF routine analysis, and was implemented on 8 April 1999. While the background signal for VHF radars is usually noise, the background signal for MF/HF radars is often coherent ionospheric echoes. Application for MF meteor studies therefore required the development of an improved detection algorithm and criteria to reject ionospheric echoes. The meteor analysis initially used receivers 1 to 5, requiring a complicated AOA estimation procedure which was often unsuccessful in resolving AOA ambiguities. Analysis from 14 September 2000 was applied using the interferometer formed by receivers 6 to 10 shown in Fig. 3, allowing for application of a technique similar to that described by Jones et al. (1998), which greatly reduced AOA ambiguities. Since the smallest effective spacing attainable is $0.6 \lambda$ the maximum unambiguous zenith angle is $56.4^{\circ}$. In most cases AOAs ambiguities can be resolved by calculating the echo height for each AOA candidate and assuming limits (70 to $140 \mathrm{~km}$ ) for valid meteor heights.

Figure 8 shows distributions of various meteor parameters, while Fig. 9 shows comparison of superposed meteor and FCA zonal winds for March 2003. A total of 8924 meteors (average 287 per day) were observed. The height distribution peaks at $105 \mathrm{~km}$ and extends from 80 to $140 \mathrm{~km}$, comparing well with previous BPMF meteor observations (e.g. Brown, 1976; Olsson Steel and Elford, 1987). The upper limit exceeds that of Tsutsumi et al. (1999) by $20 \mathrm{~km}$, which we attribute to the larger sampling range maximum used in the present study (178 km compared to $148 \mathrm{~km}$ ). The diurnal distribution shows very few meteors during the day. Those obtained are limited to heights below $100 \mathrm{~km}$ due to E-layer total reflection, as is evident from the zonal winds shown in Fig. 9. The AOAs are mostly located within $60^{\circ}$ of zenith, with the largest zenith angle being $66^{\circ}$. Less than $6 \%$ of the AOAs are detected within the main lobe of the transmit polar diagram. The decay time distribution shows good agreement with theoretical estimates obtained using CIRA86 temperature and pressure estimates (e.g. Cervera and Reid, 2000), although they show significantly more scatter than typical VHF distributions (e.g. Hocking et al., 1997; Cervera and Reid, 2000). This appears to be typical of 2-10 MHz meteor observations (e.g. Tsutsumi et al., 1999; MacDougall and Li, 2001), and may result from the effects of wind shears (e.g.
Tsutsumi et al., 1999) and turbulence (e.g. Brown, 1976), which have potentially more influence on MF/HF decay time estimates due to the longer echo durations. The velocity comparison show qualitative agreement in the overlapping height range, with the meteor estimates being larger. A similar result was observed by Cervera and Reid (1995), who found MF/FCA winds underestimated meteor wind comparisons above $90 \mathrm{~km}$. The FCA and meteor velocities clearly exhibit a diurnal variation, as expected at Adelaide in March, where the diurnal tide maximises (e.g. Holdsworth et al., 2001).

It should be stressed that the meteor analysis is applied to data collected using experimental parameters optimal for atmospheric observations, and that no parameter modification has been made to facilitate the meteor analysis. In particular, the effective sampling time of $0.4 \mathrm{~s}$ reduces the ability to detect short-lived meteors at heights above $110 \mathrm{~km}$. Thus, although the number of meteors detected is considerable lower than VHF meteor radars, Figs. 8 and 9 suggest useful observations can be made.

\section{Imaging Doppler interferometry}

Imaging Doppler interferometry (IDI) analysis (e.g. Adams et al., 1986) was introduced in August 2000, providing comparative dynamical estimates for the FCA. The fundamental assumption of IDI is that the phase information at each Doppler frequency results from a single discrete scattering location. Although some authors apply criteria to reject Doppler frequencies where this does not appear to be the case from the analysis (e.g. Adams et al., 1986; Meek and Manson, 1987). Franke et al. (1990) have shown comparable velocity estimates to the FCA are obtained without the use of rejection criteria. The IDI analysis implemented for the BPMF closely follows that of Franke et al. (1990), using antennas with similar spacings to those connected to receivers 1, 2 and 3 shown in Fig. 3. This analysis and the results are described in the accompanying paper by Holdsworth and Reid (2004).

\section{Summary and future work}

The routine analysis scheme used by the new Buckland Park MF radar has been presented. The raw data collected is simultaneously analysed by a number of different analyses for the estimation of winds, electron densities, and meteor parameters.

Figure 10 represents an attempt to compare the relative velocities shown in Tables 3 to 7 and the IDI estimates of Holdsworth and Reid (2004) to a common reference, the FCA-large velocities. As this comparison includes velocities estimated from different analyses applied over different observation periods and using different antenna configurations, it should be interpreted with some caution. The results suggest that the HDI and SCA velocities show relatively good agreement, while the DBS velocities are under- 

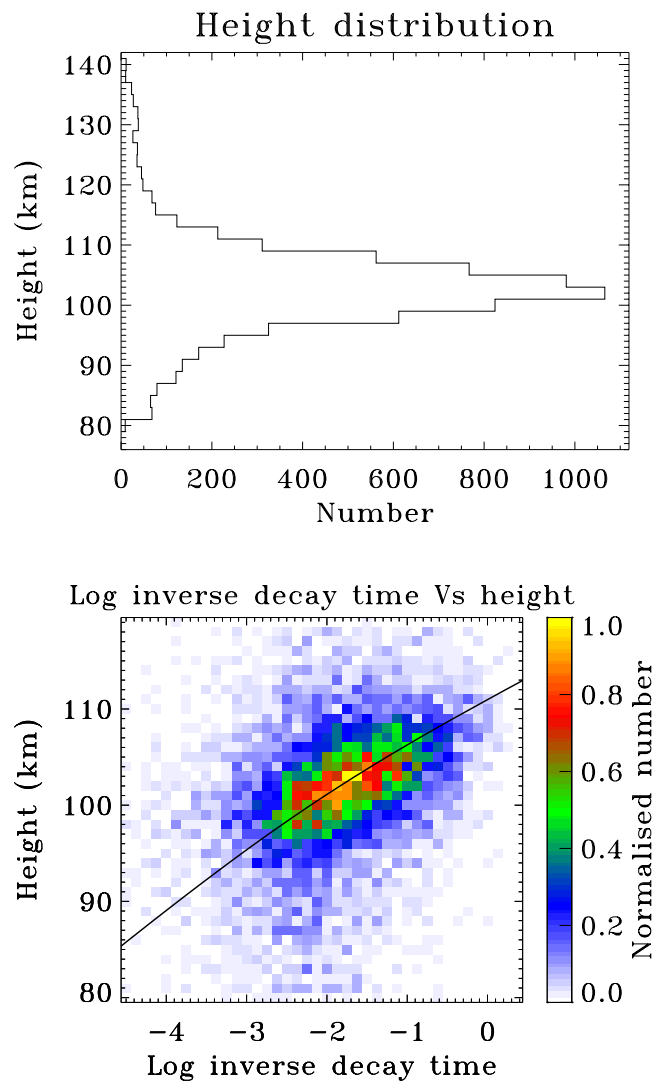
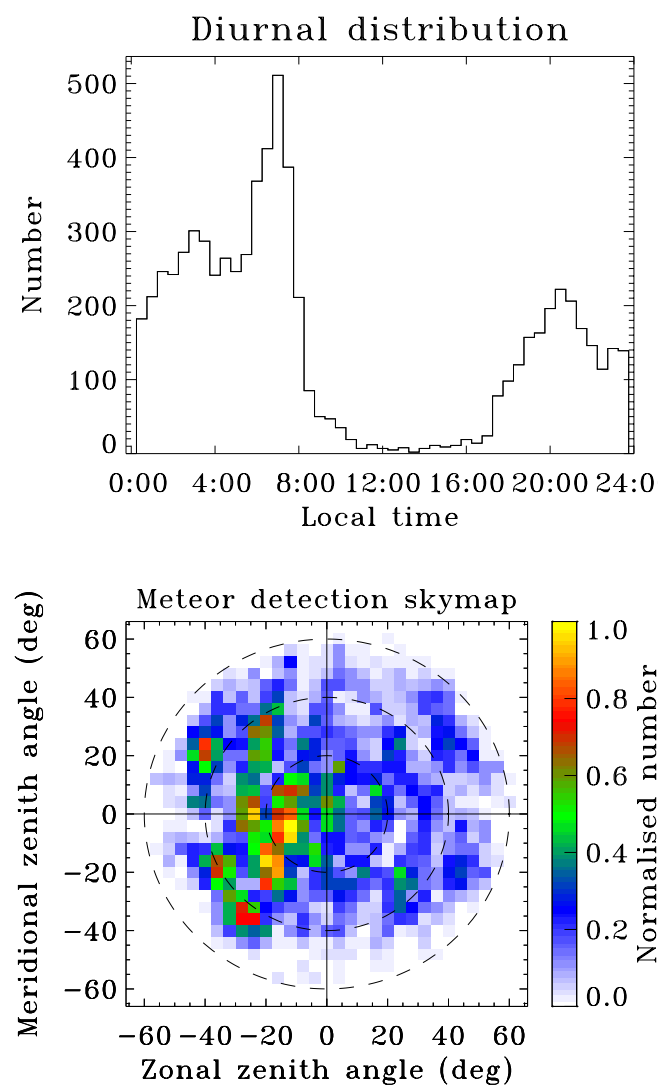

Fig. 8. Meteor distributions for March 2003: height (top left), time (top right), decay times (bottom left) and angle of arrival (bottom right). The dashed lines in the angle of arrival plot indicate zenith angles of $20^{\circ}, 40^{\circ}$, and $60^{\circ}$. The solid line in the decay time plot indicates the theoretical value estimated using CIRA temperature and pressure.
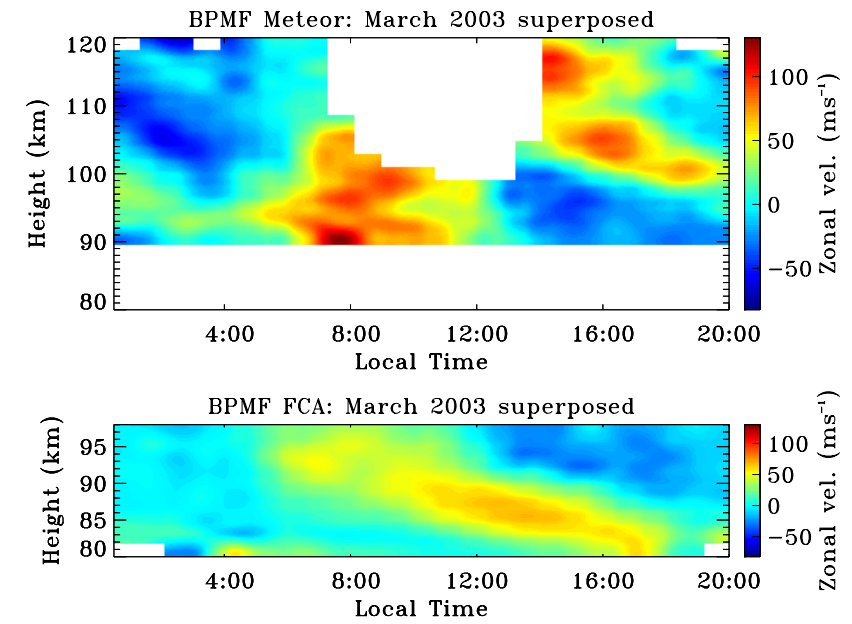

Fig. 9. Superposed velocities estimated using meteor (top) and full correlation analysis (bottom) for March 2003.

estimated. This underestimation results from EBP being biased towards zenith. HDI is able to compensate for this effect by estimating the EBP, and, therefore, provides a less biased velocity estimate than DBS. HDI is a relatively new technique, and there has been little investigation into poten-

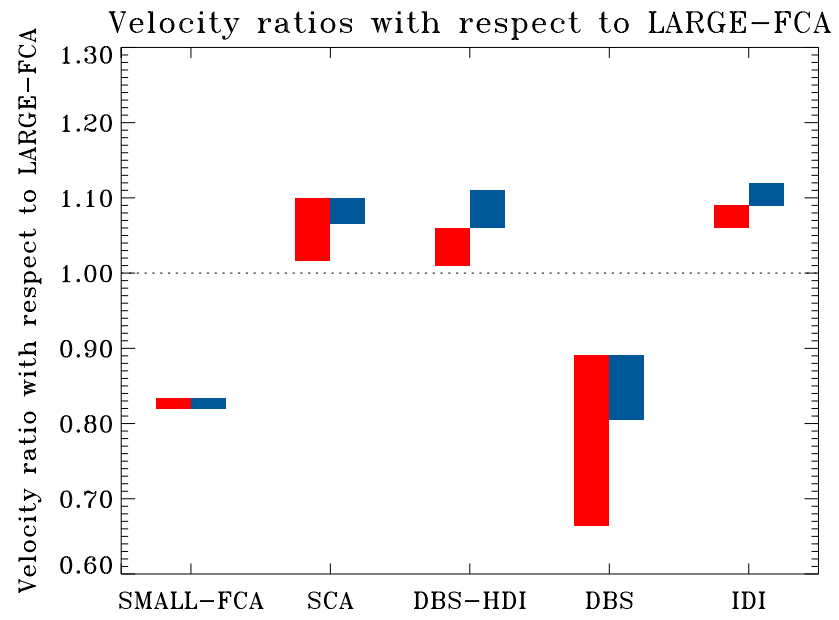

Fig. 10. Relative velocity magnitudes for various analyses with respect to "FCA-large". The red and blue bars denote the range of ratios for the zonal and meridional components, respectively.

tial biases. The SCA is expected to be independent of TSE biases, and is, therefore, expected to be a less biased velocity estimate than the FCA. Given that SCA and HDI are independent techniques whose relative magnitudes show good 
agreement and appear less biased than FCA or DBS, we feel reasonably confident that both may well be unbiased - although further investigations will be needed to confirm this is the case. On the other hand, the FCA is susceptible to the TSE, as reflected in the FCA-large velocities being approximately 5-10\% smaller than the SCA and HDI velocities. The IDI relative velocity magnitudes also show good agreement with the SCA and HDI velocities, despite predictions that the IDI velocity is overestimated in the volume scatter situation. Holdsworth and Reid (2004) suggest the agreement is due to a radial velocity threshold applied in the analysis, and that abolishing this threshold produces the volume scatter predicted overestimation.

There are a number of improvements and revised analyses intended to be implemented into the BPMF radar in the near future. One improvement involves the replacement of the DOS-based acquisition PC with a Linux PC. This will remove data transfer rate restrictions, allowing the radar to be operated with extended range maxima and reduced (or no) coherent integration. This will benefit meteor observations, with the former allowing meteors to be detected at greater ranges, and the latter improving the detectability of short-lived meteors, and allow the application of meteoroid velocity estimation techniques reliant on short effective sampling times (e.g. Cervera et al., 1997; Grant, 2004). The former may also allow improved interference rejection (e.g. Hocking, 1997b). One revised analysis we intend to implement is polarimetric DAE analysis (e.g. von Biel, 1977), which will allow daytime electron densities to be calculated to greater heights by decomposing the signals received on crossed dipoles into "elliptical" polarisation, rather than circular polarisation as used for simplicity in the current DAE analysis. The radar will continue to be used to test new analyses. A number of variations of the standard FCA are currently being tested, and preliminary results from one such analysis are described in Holdsworth and Reid (2004).

Acknowledgements. Thanks to Rupa Vuthaluru, Masaki Tsutsumi, Bob Vincent, Karen Berkefeld, Stephen Grant and Jonathan Woithe for their contributions to the work described in this paper, and Alex Didenko, Lesley Rutherford, Simon Ludbhorz, Stan Woithe and Malcolm Kirby for technical support. The Buckland Park MF radar was supported by Australian Research Council grants A69031462 and A69231890.

Topical Editor U.-P. Hoppe thanks two referees for their help in evaluating this paper.

\section{References}

Adams, G. W., Brosnahan, J. W., Walden, D. C., and Nerney, S. F.: Mesospheric observations using a $2.66 \mathrm{MHz}$ radar as an imaging Doppler interferometer, J. Geophys. Res., 91, 1671-1683, 1986.

Berkefeld, K. J.: Time domain interferometry wind estimations at mesospheric heights, Honours thesis, Univ. of Adelaide, Adelaide, Aust., 1994.

Briggs, B. H.: Radar observations of atmospheric winds and turbulence: A comparison of techniques, J. Atmos. Terr. Phys., 42, 823-833, 1980.
Briggs, B. H.: The analysis of spaced sensor records by correlation techniques, in Handbook for MAP, SCOSTEP Secr., Univ. of Ill., Urbana, 13, 166-186, 1984.

Briggs, B. H.: On radar interferometric techniques in the situation of volume scatter, Radio Sci., 30, 1, 109-114, 1995.

Briggs, B. H., Elford, W. G., Felgate, D. G., Golley, M. G., Rossiter, D. E., and Smith, J. W.: Buckland Park aerial array, Nature, 223, 5213, 1321-1325, 1969.

Brosnahan, J. W. and Adams, G. W.: The MAPSTAR Imaging Doppler Interferometer: Description and first results, J. Atmos. Terr. Phys., 55, 203-228, 1993.

Brown, N.: Radio echoes from meteor trains at a radio frequency of $1.98 \mathrm{MHz}$, J. Atmos. Terr. Phys., 33(1), 83-87, 1976.

Cervera, M. A. and Reid, I. M.: Comparison of simultaneous wind measurements using colocated VHF meteor and MF spaced antenna radar systems, Radio Science, 30, 4, 1245-1261, 1995.

Cervera, M. A. and Reid, I. M.: Comparison of atmospheric parameters derived from meteor observations with CIRA, Radio Science, 35, 3, 833-843, 2000.

Cervera, M. A., Elford, W. G., and Steel, D. I.: A new method for the measurement of meteor speeds: The pre- $t_{0}$ phase technique Radio Science, 32, 2, 805-816, 1997.

Franke, P. M., Thorsen, D., Champion, M., Franke, S. J., and Kudeki, E.: Comparisons of time- and frequency-domain techniques for wind velocity estimation using multiple-receiver MF radar data, Geophys. Res. Lett., 17, 2193-2196, 1990.

Gardner, F. F. and Pawsey, J. L.: Study of the ionospheric D-region using partial reflections, J. Atmos. Terr. Phys., 3, 321-344, 1953

Golley, M. G. and Rossiter, D. E.: Some tests of methods of analysis of ionospheric drift records using an array of 89 aerials, J. Atmos. Terr. Phys., 32, 1215-1233, 1970.

Grant, S. I.: Radar studies of meteors at medium frequency, Ph. D. thesis, University of Adelaide, Australia, 2004.

Hocke, K. and Igarashi, K.: Variability and anisotropy of mesospheric wind spectra Geophys. Res. Lett., 24, 22, 2725-2728, 1997.

Hocking, W. K.: System design, signal-processing procedures, and preliminary results for Canadian (London, Ontario) VHF atmospheric radar Radio Sci, 32, 2, 687-706, 1997a.

Hocking, W. K.: Recent advances in radar instrumentation and techniques for studies of the mesosphere, stratosphere and troposphere, Radio Sci, 32, 6, 2241-2270, 1997 b.

Hocking, W. K., Thayaparan, T., Franke, S. J.: Method for statistical comparison of geophysical data by multiple instruments which have differing accuracies, Adv. Space Res., 27(6-7), 1089-1098, 2001.

Hocking, W. K., Thayaparan, T., and Jones, J.: Meteor decay times and their use in determining a diagnostic mesospheric temperature-pressure parameter: methodology and one year of data Geophys. Res. Lett., 24, 23, 2977-2980, 1997.

Holdsworth, D. A.: Spatial correlation analysis revisited: Theory, and application using "radar backscatter model" data, Radio Sci., 34, 3, 629-642, 1999a.

Holdsworth, D. A.: The influence of instrumental effects upon the full correlation analysis, Radio Sci., 34, 3, 643-656, 1999b.

Holdsworth, D. A. and Reid, I. M.: A simple model of atmospheric radar backscatter: Description and application to the full correlation analysis of spaced antenna data, Radio Sci., 30, 4, 1263 1280, 1995a.

Holdsworth, D. A. and Reid, I. M.: Spaced antenna analysis of atmospheric radar backscatter model data, Radio Sci., 30, 5, $1417-$ $1433,1995 b$ 
Holdsworth, D. A. and Reid, I. M.: An investigation of biases in the full correlation analysis technique, Adv. Space Res., 20, 6, 1269-1272, 1997.

Holdsworth, D. A. and Reid, I. M.: The Buckland Park meteor radar - description and initial results, Proceedings "Workshop on Applications of Radio Science”, Leura, Australia, 2002.

Holdsworth, D. A. and Reid, I. M.: Comparisons of full correlation analysis (FCA) and imaging Doppler interferometry (IDI) winds using the Buckland Park MF radar, Ann. Geophys., 11, 3829$3842,2004$.

Holdsworth, D. A., Vincent, R. A., and Reid, I. M.: Mesospheric turbulent velocity estimation using the Buckland Park MF radar, Ann. Geophys., 19, 8, 1007-1017, 2001.

Holdsworth, D. A., Vuthaluru, R., Vincent, R. A., and Reid, I. M.: Differential absorption measurements of mesospheric and lower thermospheric electron densities using the Buckland Park MF radar, J. Atmos. Solar Terr. Phys, 64, 18, 2029-2042, 2002.

Jones, J., Webster, A. W., and Hocking, W. K.: An improved interferometer design for use with meteor radars, Radio Science, 33, 1, 55-66, 1998.

Kawano, N., Tahara, Y., and Fukao, S.: Comparison of wind estimation errors for the spaced antenna technique: A case study for the MU radar, Radio Sci., 37, 5, doi:10.1029/2000RS002533, 2002.

Kudeki, E. and Woodman, R. F.: A post-statistics steering technique for MST radar applications, Radio Sci., 25, 4, 591-594, 1990.

Kudeki, E., Sürücü, F., and Woodman, R. F.: Mesospheric wind and aspect sensitivity measurements at Jicamarca using radar interferometry and poststatistics steering techniques, Radio Sci., 25, 4, 595-612, 1990.

Lesicar, D.: Study of the structure of partial reflection radar scatterers and their application in atmospheric measurements, Ph.D. thesis, University of Adelaide, Adelaide, 1993.

Lesicar, D. and Hocking, W. K.: Studies of the seasonal behavior of the shape of mesospheric scatterers using a $1.98 \mathrm{MHz}$ radar, J. Atmos. Terr. Phys., 54, 295-309, 1992a.

Lesicar, D., Hocking, W. K., and Vincent, R. A.: Comparative studies of scatterers observed by MF radars in the polar summer hemisphere mesosphere, J. Geophys. Res., 97, 887-897, 1992b.

MacDougall, J. W. and Li, X.: Meteor observations with a modern digital ionosonde, J. Atmos. Terr. Phys., 63, 2-3, 135-141, 2001.

Manson, A. H., Meek, C. E., Massebeuf, M., Fellous, J. L., Elford, W. G. Vincent, R. A., Craig, R. L., Roper, R. G., Avery, S. K., Balsley, B. B., Fraser, G. J, Smith, M. J., Clark, R. R., Kato, S., and Tsuda, T.: Mean winds in the upper atmosphere $(70-110 \mathrm{~km})$ from the global radar network: comparisons with CIRA 72, and new rocket and satellite data, Adv. Space Res, 7, 10 143-10 153, 1987.

Manson, A. H., Meek, C. E., Fleming, E., Chandra, S., Vincent, R. A., Phillips, A., Avery, S. K., Fraser, G. J, Smith, M. J., Fellous, J. L., and Massebeuf, M.: Comparisons between satellite derived gradient winds and radar derived winds from CIRA-86, J. Atmos. Sci., 48, 3, 411-428, 1991.
Meek, C. E.: Triangle size effect in spaced antenna wind measurements, Radio Sci., 25, 4, 641-648, 1990.

Meek, C. E. and Manson, A. H.: Mesospheric motions observed by simultaneous Medium-frequency Interferometer and Spaced Antenna experiments, J. Geophys. Res., 92, 5627-5639, 1987.

Nakamura, T., Tsuda, T., and Fukao, S.: Mean winds at $60-90 \mathrm{~km}$ observed with the MU radar $\left(35^{\circ} \mathrm{N}\right)$, J. Atmos. Terr. Phys., 58, 6, 655-660, 1996.

Olsson Steel, D. I. and Elford, W. G.: The height distribution of radio meteors: observations at $2 \mathrm{MHz}$, J. Atmos. Terr. Phys., 49, 243-258, 1987.

Reid, I. M., Vandepeer, B. G. W., Dillon, S. C., and Fuller, B. M.: The new Adelaide medium frequency Doppler radar, Radio Sci., 30, 4, 1177-1189, 1995.

Røyrvik, O.: Spaced antenna drift at Jicamarca, mesospheric measurements, Radio Sci., 18, 3, 461-476, 1983.

Thorsen, D., Franke, S. J., and Kudeki, E.: A new approach to MF radar interferometry for estimating mean winds and momentum flux, Radio Sci., 32, 2, 707-726, 1997.

Tsutsumi, M., Holdsworth, D. A., Nakamura, T., and Reid, I. M.: Meteor observations with an MF radar, Earth, Planets, Space, 51, 691-699, 1999.

Vandepeer, B. G. W.: A new MF Doppler radar for upper atmospheric research, Ph.D. thesis, Univ. of Adelaide, Adelaide, Aust., 1993.

Vandepeer, B. G. W. and Reid, I. M.: Some preliminary results obtained with the new Adelaide MF Doppler radar, Radio Sci., 30, 4, 1191-1203, 1995.

Vincent, R. A. and Reid, I. M.: HF radar measurements of mesospheric gravity wave momentum fluxes, J. Atmos. Sci., 40, 1321-1333, 1983.

Vincent, R. A. and Lesicar, D.: Dynamics of the equatorial mesosphere: First results with a new generation partial reflection radar, Geophys. Res. Lett., 18, 5, 825-828, 1991.

Vincent, R. A., May, P. T., Hocking, W. K., Elford, W. G., Candy, B. H., and Briggs, B. H.: First results with the Adelaide VHF radar: Spaced antenna studies of tropospheric winds, J. Atmos. Terr. Phys., 49, 353-366, 1987.

von Biel, H. A.: The phase switched correlation polarimeter - a new approach to the partial reflection experiment, J. Atmos. Terr. Phys., 39, 769-778, 1977.

Vuthaluru, R., Vincent, R. A., Holdsworth, D. A., and Reid, I. M.: Collision frequencies in the D-region, J. Atmos. Solar Terr. Phys, 64, 18, 2043-2054, 2002.

Woodman, R. F. and Guillen, A.: Radar observations of winds and turbulence in stratosphere and mesosphere, J. Atmos. Sci., 31, 493-505, 1974. 